\title{
A programmable proximity-contact sensor to detect location or locomotion of animals
}

\author{
REX L. CLARKE, ROBERT F. SMITH, and DON R. JUSTESEN \\ USVA Medical Center, Kansas City, Missouri
}

\begin{abstract}
A simple electronic device to detect location or to track locomotion of small animals is described. The device is based on an antenna effect-it responds to a change in capacitance as the body of the animal passes between an antenna and one of a series of contact plates. A digital address system is incorporated that is capable of electronically polling the contact plates according to instructions from a data-acquisition system (DAS). Any DAS capable of generating an address of two bits or more and of reading a one-bit data line may be employed. The device is ideal for mazes or other apparatus in which the exclusion of stereotypical behaviors is desired.
\end{abstract}

Numerous mechanical, electromechanical, and electronic sensors have been devised to measure general activity. Among them are sensors based on ultrasound (Akaka \& Houck, 1980), Doppler radar (Martin \& Unwin, 1980), body resistance (Tarpy \& Murcek, 1984), and infrared (IR) detection (Clarke, Smith, \& Justesen, 1985). Only one noncommercial device has been designed to measure, independently and simultaneously, locomotor and stereotypical components of activity (Clarke, Smith, \& Justesen, 1988). Based on IR detection, the device uses electronic latches and logic circuits to differentiate components of behavior.

Conventional IR-photocell activity monitors, like most other techniques of measuring activity, provide incremental rather than discrete sampling of behavior. Activity monitors, whatever the mode of measurement, sometimes provide spurious information that may obscure or confound results of experiments. For example, the stereotypical activities of a rat in a maze (grooming, scratching, rearing, etc.) are usually irrelevant to an investigator's objective. Often what is needed is a discrete sampling of some substantial and determinable changes in location over time, that is, locomotion. An array of IR beams connected to latch circuits can be used to differentiate stereotypical from locomotor behaviors in these situations. When large areas must be monitored, however, the use of latched IR-photocell activity monitors can be very expensive.

A cost-effective alternative is found in the electronic device known as a "contact," or "proximity," switch. There are many versions of these devices, which operate by different principles. The contact, or proximity, switch with the simplest sensor element detects a change in capacitance between an antenna wire and a metal plate

Our work was supported by funds from the Research Service of the U.S. Department of Veterans Affairs. Address correspondence to R. L. Clarke, Behavioral Radiology Laboratories, Research Service 151, USVA Medical Center, Kansas City, MO 64128. as an animal approaches or touches the plate (antenna effect). A series of such switches, when polled in sequence, can provide a means for detecting an animal's location without confounding by stereotypical behaviors. Integrated over time, the sequence of locations will provide an undiluted record of the animal's locomotor behavior.

Despite conceptual and electronic simplicity, circuits to detect locomotor behavior of small animals by bodycapacitance sensing are uncommon in the literature on instrumentation. This lack is remarkable because many standard electronic reference texts provide a variety of circuits that employ capacitive-sensing principles. Some circuits would appear to be readily adaptable to detection of location or, given scanning and memory storage, of locomotion (see Graf, 1985, and Lancaster \& Berlin, 1988).

A possible reason for the neglect of contact-proximity sensors based on body capacitance became evident after some representative circuits were breadboarded. Simply put, however well they may work in sensing the pressure or presence of the human finger, the basic circuits described in the literature did not work well with small animals.

In this report, we describe the circuit of a novel, inexpensive, and reliable circuit that detects a small animal that is proximal to or in contact with a metal plate. The circuit operates in a digital-all or none-fashion. Developed from a synthesis of concepts found in contemporary antitampering and burglar-alarm circuitry (see Carr, 1988), the detector appears to be immune to false triggering and hypersensitivity problems, which plagued simple contact-proximity circuits that the senior author breadboarded from diagrams in the electronic reference texts.

\section{The Programmable Contact-Proximity Detection Circuit}

A 555 IC timer (Figure 1) is configured as an astable multivibrator with a dual-polarity current source to obtain a symmetrical output about the zero axis. The output of the multivibrator is applied to a balanced-bridge cir- 


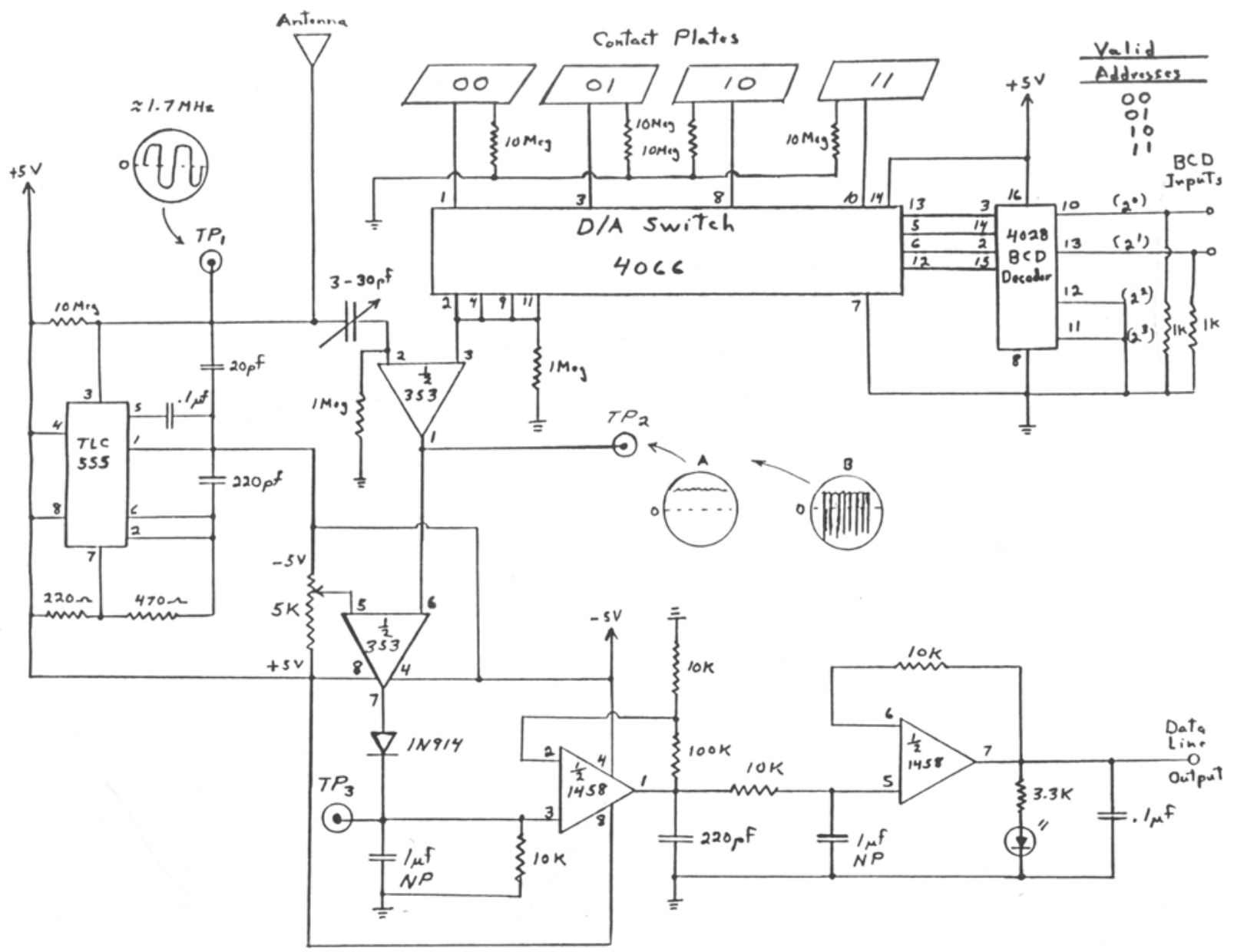

Figure 1. A schematic diagram of the basic proximity-contact detector. If the full BCD byte is used, as many as $\mathbf{1 0}$ contact plates can be addressed if additional digital-to-analog switches are used. When the 10-Mn swamping resistors shown in the diagram are attached to the contact plates, the device will function only as a contact detector.

cuit. Because the same signal is applied to both legs of the bridge, neither exactness nor drift of frequency is critical within wide limits; therefore, the circuit is not temperature-sensitive.

The bridge consists of a variable capacitor and a 1-M $\Omega$ resistor, which form two legs, and an antenna wire, a metal plate, and a 1-Mn resistor, which form the other two legs. The antenna wire and metal plate found in one leg of the bridge serves as a capacitor of a few picofarads that is balanced by the variable capacitor in the opposite leg. The exact capacitance depends on the proximity of the antenna wire to the plate, the size of the plate, and the length of the wire.

Any error signal from an unbalanced bridge is fed to a differential amplifier (one half of a 353 IC). The output from the differential amplifier passes to an inverting comparator (the second half of the 353 IC) that determines the voltage at which an output can occur. From the comparator, the error signal passes to a detector diode (1N914), a low-pass amplifier (one half of a 1458 IC), and to an active, low-pass filter (consisting of the second half of the 1458 IC). The output of this filter drives an LED that gives a visual indication of the contact between an animal and a sensor plate and is the data line to a dataacquisition system (DAS).

The digital section of this programmable contact-switch device consists of a quad digital-to-analog switch (IC 4066) and a BCD decoder (IC 4028). One pole of each of the four electronic switches in the 4066 is common and is attached to the differential amplifier. The other pole of each switch is attached to one of a series of metal plates, which are marked 00,01,10, and 11 in Figure 1 (their binary addresses). These plates are connected, one at a time, to the differential amplifier by an address from the DAS. As each is connected, the DAS checks the data line for the presence of a 1 or a $0(5$ or $0 \mathrm{~V})$.

The control lines for the electronic switches of the 4066, the IC that determines which of the four metal plates is in contact with the differential amplifier, are coupled from a BCD-to-decimal decoder (4028 IC). Because only four 
contact plates are used in the example in Figure 1, only two of the BCD inputs from the DAS are needed (the valid addresses correspond to the binary numbers on the appropriate plate).

All BCD inputs are connected to ground directly or through $1,000-\Omega$ resistors. These connections ensure that when there is no address to the BCD inputs of the 4028 , a pseudoaddress of 00 is constantly imposed (i.e., Plate 00 maintains continuity with the differential amplifier). This arrangement makes it simple to balance the bridge (the normal condition that the presence of an animal on a contact plate upsets) by setting the variable capacitor to a value equal to the capacitance between Contact Plate 00 and the antenna wire.

In Figure 1, each contact plate is shown connected directly to chassis (and earth) ground through a 10-M $\Omega$ resistor (which is in parallel with the bridge $1-\mathrm{M} \Omega$ resistor when that plate is activated). These swamping resistors suppress transients and decrease the sensitivity of the device so that it functions as a contact rather than as a proximity sensor. So long as these resistors are 10-M $\Omega$ or less, they may be connected to any locus on the plate, to the line going to the D/A switch, or to the $4066 \mathrm{IC}$ itself. If higher values of resistance are used, the exact placement of the resistors may become critical, requiring that resistances be determined empirically.

Balancing the variable capacitor in the bridge circuit is best accomplished with an oscilloscope. Without an input of address and without an animal on the 00-contact plate, Test Point 2 (TP2) should display a waveform that varies between that labeled $A$ (subcritical) and that labeled B (critical) in Figure 1 as the bridge-balance capacitor is adjusted. The proper operational setting is found at the transition from subcritical to critical, but slightly in the subcritical direction. Next, an animal should be placed in contact with Plate 00 , and the DC voltage should be noted at Test Point 3 (TP3). This voltage approximates that which the $5 \mathrm{~K}$ comparator potentiometer should have on its center tap. The potentiometer should now be adjusted slowly, with an animal on the Contact Plate 00, until the LED illuminates. The remainder of the plates should be checked by manually inserting the appropriate $\mathrm{BCD}$ address (i.e., apply $5 \mathrm{~V}$ to or ground the appropriate line) and touching the corresponding contact plate while noting the state of the LED.

The contact sensor plates used in this device are flexible in the sense that they can differ considerably from each other in size and geometry (they can be conformed to any desired shape). With large differences in size, however, some degree of suboptimal adjustment of the bridge capacitor will be required. This adjustment is needed because the antenna-wire-to-contact-plate capacitance will change slightly as the DAS steps the system across the plates. If the bridge capacitor were left at its optimal setting, the system might see some plates as critical (i.e., as a rat making contact) when the rat is not actually present. A suboptimal adjustment of the bridge capacitor results in a decline in sensitivity that can be compensated for by an increase in the values of the swamping resistors (they do not need to have identical values).

If this device is used in the proximity mode, the contact plates, for obvious reasons, cannot be close to each other. The exact configuration of the detector when used in this mode must be arrived at empirically. The pertinent variables are the length of the antenna wire, the proximity of the wire to the plates, the separation between the contact plates, and the value(s) of the swamping resistors.

\section{Discussion}

General activity is one of the most common measures of nonoperant animal behavior. In many experiments, it may not only be unnecessary but undesirable to attempt to partition behavior into its locomotor and stereotypic components. Such partitioning, however, can sometimes give unexpected perspectives to a study by eliminating extraneous data.

The researcher always confronts the rule that says that only after an experiment has concluded and the data have been analyzed will it be clear exactly what should have been measured (or excluded from measurement). Convenience is always a tacit but forceful factor in deciding how and what data will be collected. Once the relatively simple electronic device described here is constructed, it takes only a few minutes with a tube of adhesive and a pair of tin-snips (or a paper cutter, if aluminum sheeting is used) to construct and position a contact sensor of whatever geometry is desired. A series of such sensors, in the form of easily shaped, thin-metal plates, when scanned in rotation by any of the several DASs available for microcomputers that are capable of binary input and output, can form an effective location, or locomotion, detector. For example, with a typical DAS (the Isaac-91A, the Cyborg Corp., Newton, MA), each location number of the contact plates is read out in sequence (by a for-next loop) over the binary input/output lines from the Cyborg to the contact detector. The active location is sampled via the detector-output data line to the binary (Schmitt-trigger) input of the Cyborg. The location is assigned a 1 (if the addressed plate is occupied) and placed in its proper binary-word location (e.g., 1000). All four locations are sampled, and a binary word is constructed that the operator may translate to a decimal value, convert to a text value, or store in its original form. Given four locations, any single location or any two locations may be active. There are 10 possible binary values - that is, the rat may be on any one of the four contact plates or on any two adjoining plates. An outer for-next loop is used to determine the sample-unit time, the number of samples, the data array, the data treatment, and the data storage.

We emphasize that the basic circuit in Figure 1 can easily be elaborated by additional contact plates as required to provide the requisite degree of resolution (i.e., detection of small increments of locomotion). The single 4028 BCD-to-decimal decoder can handle up to 10 contact plates (i.e., each BCD byte can handle up to 10 plates- 
addresses 0000-1001), if additional D/A switches (IC 4066s) are provided.

In conclusion, we note several caveats: The contactproximity sensing portion of our device was designed to detect animals with the body mass of an adult rat $(\sim 250-$ $500 \mathrm{~g}$ ). To detect a smaller animal, such as a mouse, it will be necessary to increase the output voltage from the multivibrator or to increase its frequency, or both. These adjustments are easy to do if the Radio Shack TLC 555 has been used or is substituted as the multivibrator IC. The Vdd can be increased to +9 volts and the Vss to -9 volts (the voltages must mirror one another). The frequency may be increased to $2 \mathrm{MHz}$ by substituting a capacitor of lower value for the $220-\mathrm{pF}$ capacitor found between Pins 1 and 6 of the 555 IC. If the former stratagem is used, we also recommend that the same voltages be used as Vdd and Vss on the 353 IC (differential amplifier and comparator). Other changes that might be considered are combinations of the adjustments that increase sensitivity and that were mentioned in the use of the device in the proximity-detection mode.

Within limits, urine and fecal contamination of the contact plates is not a problem except in those instances in which urine short-circuits closely adjacent plates. If an animal is on either plate, it will register on both plates until the urine dries. However, if the separation between the plates is extended by at least the width of the body of the experimental animal, short-circuiting is unlikely.
We note, finally, that our device should not be used in proximity to large masses of metal. Grounded masses of metal, such as drain pipes, are particularly prone to interfere.

\section{REFERENCES}

Akaka, W. H., \& Houck, B. A. (1980). The use of an ultrasonic monitor for recording locomotor activity. Behavior Research Methods \& Instrumentation, $12,514-516$

CARR, J. J. (1988). IC users casebook. Carmel, IN: Howard W. Sams

Clarke, R. L., Smith, R. F., \& Justesen, D. R. (1985). An infrared device for detecting locomotor activity. Behavior Research Methods, Instruments, \& Computers, 17, 519-525.

Clarke, R. L., Smith, R. F., \& Justesen, D. R. (1988). An infrared device to monitor discrete ambulatory and stereotypic behaviors. Behavior Research Methods, Instruments, \& Computers, 20, 404-407.

GraF, R. F. (1985). The encyclopedia of electronic circuits. Blue Ridge Summit, PA: TAB Books.

LANCASTER, D., \& BerLIN, H. M. (1988). CMOS casebook (2nd ed.). Carmel, IN: Howard W. Sams.

MARTIN, P. H., \& UNwIN, D. M. (1980). A microwave doppler radar activity monitor. Behavior Research Methods \& Instrumentation, 12 , 517-520.

TARPY, R. M., MURCEK, R. J. (1984). An electronic device for detecting activity in caged rodents. Behavior Research Methods, Instruments, \& Computers, 16, 383-387.

(Manuscript received October 28, 1991; revision accepted for publication May 26, 1992.) 